\title{
Simulation of Counter-Current Imbibition in SRVs of Tight Oil Reservoir
}

\author{
Zhongyi Xu, Linsong Cheng, Renyi Cao, and Sidong Fang
}

\begin{abstract}
During the process of tight oil exploration, counter current imbibition effect is significantly different due to the presence of complex fracture network and flow characteristics in tight oil reservoir. To simulate counter-current imbibition in fractured tight oil reservoir properly. PEBI grids are used to match the complex fracture network, natural fractures and matrix are idealized as dual-porosity medium, rate of mass transfer of imbibition between matrix and fractures is treated as source or sink term in dual porosity model. A new semi analytical model for the calculation of mass transfer function of counter-current imbibition in the presence of complex fracture network is presented by using radial integration boundary element method (RIBEM), In addition, to reflect the flow characteristics of tight oil, relative permeability and capillary pressure curve, which have considered the effect of boundary layer, has also used in the mass transfer model. Besides, with a field example from tight oil reservoir, we show the capacity and practical use of the model. From simulated results, it is concluded that counter-current imbibition place an important role on the early and middle stage of exploration and the existence of boundary layer makes the contribution of imbibition to oil production greatly reduced.
\end{abstract}

Index Terms-Tight oil reservoir, boundary layer, counter current imbibition, RIBEM.

\section{INTRODUCTION}

As shown in Fig. 1. the counter-current imbibition is the main recovery mechanism in the tight oil production process, different from ordinary imbibition, the exist of fracture network make the contact area of water between fracture and matrix increase dramatically, on the other hand, the micro-scale throat in tight porous media make the imbibition different from ordinary porous media. Current numerical simulation study on imbibition have not consider the exist of fracture network and flow characteristic in tight formation. therefore, the purpose of this article is to model the counter-current imbibition in real tight oil formation, for complex fracture network, PEBI grids are used to match fracture, for flow characteristic in tight oil formation, boundary layer theory is applied in the calculation of imbibition diffusion coefficient. In the end, with a field example from changing, we show the practical use of the proposed model to evaluate well performance of counter

Manuscript received December 13, 2017; revised March 20, 2018.

Zhongyi Xu, Linsong Cheng, Renyi Cao, Sidong Fang are with the College of Petroleum Engineering, China University of Petroleum, Beijing, China (e-mail: 13810421945@163.com, 1scheng@cup.edu.cn, Caorenyi@126.com, jbwolfgeng@163.com) current imbibition in tight reservoirs.

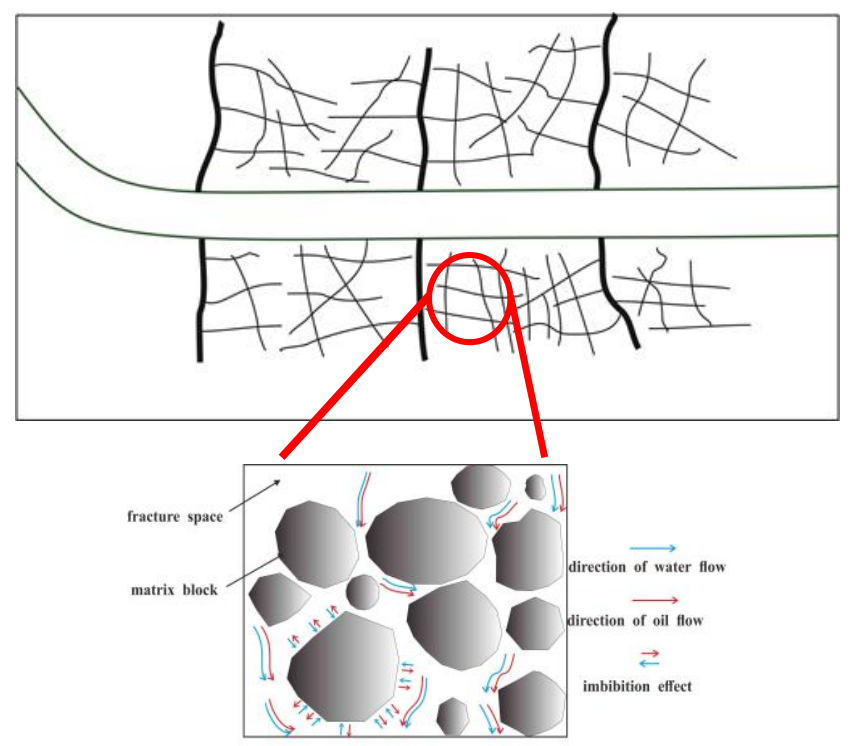

Fig. 1. Schematic of counter-current imbibition in the multi-staged fracturing horizontal well tight oil reservoir.

\section{Fracture Network Description Method}

The technologies of horizontal well drilling and hydraulic are widely used to make the commercial development of tight oil reservoir. The combination of these technologies creates stimulated reservoir volume (SRV) near wellbore area. In SRV areas complex fracture network consist of hydraulic fracture and popped nature fractures [1].

The conductivity of hydraulic fracture is much higher than nature fracture and it is important for the fluid flow and distribution, so the hydraulic fracture need to be described explicitly, as for nature fracture, although conductivity is small, the number is enumerous.so the role of nature fracture is also very important, but if the nature fractures are described explicitly, it's not only difficult to accomplish but also to reduce the efficiency of simulation, so in this article dual porosity model is used to represent natural fracture and matrix block media.in order to match the explicit hydraulic fracture, PEBI grids are used [2].

The example case of generated PEBI grids are shown in Fig. 2, from Fig. 2 (a), grid density near hydraulic fracture is higher, demonstrate the fractures in SRV area are highly developed. As shown in Fig. 2 (b), PEBI grids are formed on either side of explicit fracture, as shown in Fig. 2 (c), at the interaction point, PEBI grids are formed in each side of interact fractures, fluid flows direction are dynamically determined by node pressure. 


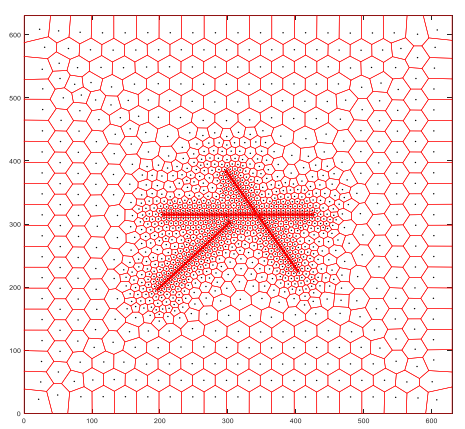

(a) PEBI mesh generation for fitting explicit fractures

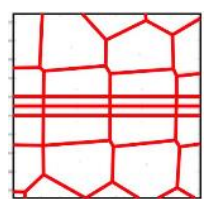

(b) schematic of PEBI grids near explicit fractures

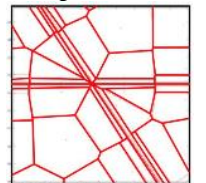

(c) schematic of PEBI grids when fractures interact

Fig. 2. Schematic of PEBI mesh for complex fractures network.

\section{MATHEMATICAL MODEL}

\section{A. Two-Phase Flow Model}

When fracturing fluid or water flows in the fracture network, counter-current imbibition occurs under the driving force of capillary pressure, two-phase flow model considering imbibition obeys the following assumptions:

(1) The whole flow medium is made of matrix blocks and the fracture around them.

(2) Average water saturation is used to represent the average oil and water change into each matrix block.

(3) Governing equation of counter-current imbibition obey the diffusion form equation.

(4) During the counter current imbibition process, water saturation changes in the fracture are the boundary condition of each matrix block.

The whole nature fracture and matrix are presented by dual porosity model, all the fluid flow in fracture medium obey the Darcy law, mass transfer of imbibition act as sink and source term in the $\mathrm{Eq}(1-2)$.

$$
\begin{aligned}
& \nabla \cdot\left(K \nabla p_{o}\right)+q_{w}+q_{i m o}=\mu_{o} C_{t} \varphi \frac{\partial p_{o}}{\partial t} \\
& \nabla \cdot\left(K \nabla p_{w}\right)+q_{w}+q_{i m w}=\mu_{w} C_{t} \varphi \frac{\partial p_{w}}{\partial t}
\end{aligned}
$$

Here, $\mathrm{K}$ is permeability of nature fracture, $p_{\mathrm{o}}$ and $p_{\mathrm{w}}$ are pressure of oil and water phase, $q_{\mathrm{o}}$ and $q_{\mathrm{w}}$ are sink and source term, $q_{\mathrm{imo}}$ and $q_{\mathrm{imw}}$ are mass transfer term of imbibition, $\mu_{\mathrm{o}}$ and $\mu_{\mathrm{w}}$ are viscosity of oil and water, $\varphi$ is porosity, $C_{\mathrm{t}}$ is total compressibility, $t$ is time.

As long as to accurately describe the imbibition sink and source term, we can find out the influence of the volume fracturing development process.

\section{B. Boundary Layer in Tight Oil Formation}

Imbibition occurs in tight porous media , throat of tight formation is tiny and reached up to Nano-scale, based on the theory of Cao Renyi [3], under this scale boundary layer has a great effect on fluid flow in tight porous media.as shown in Fig. 3, the exist of boundary layer decrease the effect throat radius and make the volume of flowable fluid dramatically decrease, in order to quantitatively characterize the influence of boundary layer on fluid flow, Tian [4] published the expression of boundary layer thickness:

$$
h_{i}=\left\{\begin{array}{lr}
r_{i} 0.25763 e^{-0.261 r_{i}}(\nabla P)^{-0.419} \mu, \nabla P<1 M p a / m \\
r_{i} 0.25763 e^{-0.261 r_{i}} \mu, & \nabla P>1 M p a / m
\end{array}\right.
$$

where $r_{i}$ is the radius of pore $i, h_{i}$ is boundary layer thickness of $r_{i} \cdot \nabla p$ is pressure gradient, $\mu$ is viscosity of fluid.

From the expression, boundary layer is the function of throat radius, viscosity and pressure gradient, based on Cao Renyi [3], matrix permeability of tight formation can be express as follows:

$$
K_{m}=\frac{\varphi_{m} \sum_{i=1}^{i=n}\left[\exp \left(-\frac{\left(r_{i}-r_{\text {avg }}\right)}{2 \sigma^{2}}\right)^{2}\left(r_{i}-h_{i}\right)^{4}\right]}{8 \tau \sum_{i=1}^{i=n}\left[\exp \left(-\frac{\left(r_{i}-r_{\text {avg }}\right)}{2 \sigma^{2}}\right)^{2}\left(r_{i}-h_{i}\right)^{2}\right]}
$$

where $K_{\mathrm{m}}$ is permeability of matrix, $r_{\mathrm{avg}}$ is average radius of pores. $\varphi_{\mathrm{m}}$-porosity of matrix, $\tau$ is tortuosity.

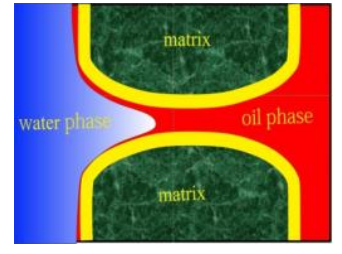

(a)throat with boundary layer

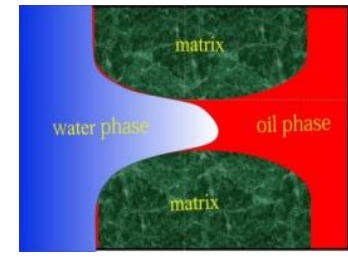

(b)throat without boundary layer
Fig. 3. Comparison of throat with or without boundary layer.

In water wet formation, Imbibition is a two phase flowing process, the relative permeability and capillary pressure are the determine parameter for counter-current imbibition, these parameters are also effected by the boundary layer, therefor the analytical solution of capillary pressure is shown in $\mathrm{Eq}(6)$

$$
\begin{gathered}
r^{\prime}=r-h \\
p_{c}^{\prime}=\frac{2 \sigma_{o w} \cos \theta_{o w}}{r^{\prime}}
\end{gathered}
$$

Here, $r$ ' is effective pore radius. $p_{c}$ ' is capillary pressure of pores with boundary layer. $\sigma$ is interfacial tension, $\theta$ is contact angle.

Based on Purcell [5] and Tian [4], Eq (7) and Eq (8) are analytical expressions for the relative permeability curve considering boundary layer.

$$
\begin{gathered}
K_{r w}\left(S_{w i}\right)=\frac{\int_{S_{w c}}^{S_{w i}} \frac{1}{\left(p_{c}^{\prime}\right)^{2}} d S}{\int_{S_{w c}}^{1-S_{o r}} \frac{1}{\left(p_{c}^{\prime}\right)^{2}} d S} \\
K_{r o}\left(S_{w i}\right)=\frac{\int_{S_{w c}}^{1-S_{o r}} \frac{1}{\left(p_{c}^{\prime}\right)^{2}} d S}{\int_{S_{w c}}^{1-S_{o r}} \frac{1}{\left(p_{c}^{\prime}\right)^{2}} d S}
\end{gathered}
$$

Here, $K_{r o}$ and $K_{\mathrm{rw}}$ are relative permeability of oil and water, $S_{\mathrm{wc}}$ is irreducible water saturation, $S_{\mathrm{or}}$ is residual oil saturation, $S_{\mathrm{wi}}$ is water saturation when water entries into the throat whose radius is $r_{\mathrm{i}}$ 


\section{Mass Transfer Model}

In matrix block system, change in matrix is completely controlled by capillary pressure and the counter current imbibition process is described as a nonlinear diffusion equation [6] which is described in Eq.(9).

$$
\frac{\partial}{\partial x_{i}}\left(D\left(S_{w}\right) \frac{\partial S_{w}}{\partial x_{i}}\right)=\phi \frac{\partial S_{w}}{\partial t}
$$

$D\left(S_{w}\right)$ is called capillary diffusion coefficient and is express as Eq.(10)

$$
D\left(S_{w}\right)=\phi\left(\frac{1}{\lambda_{o}}+\frac{1}{\lambda_{w}}\right)\left(\frac{\partial p_{c}^{\prime}}{\partial S_{w}}\right)^{-1}
$$

$\lambda_{o}$ and $\lambda_{w}$ are mobility, $P_{c}$ is capillary pressure.

In order to take into account the flow characteristics of tight reservoir, effective pore radius is used to calculate the capillary diffusion coefficient. The mobility and relative permeability is also replaced by $\mathrm{Eq}(6-8)$ in mobility term of capillary diffusion coefficient. $D\left(S_{w}\right)$ in general is a bell shaped function of water saturation, as shown in Fig. 4. In order to solve this nonlinear equation in matrix block, successive steady state substitution method is applied to handle the capillary diffusion coefficient, the real capillary diffusion coefficient is treated as multi-segment piecewise function, the saturation distribution in the matrix is simplified as the average water saturation, during a very short time can be considered as constant and is also can be simplified as a constant and denote as $D_{e}$, shown in Fig. 4. The change of parameter is calculated based on new calculated average water saturation during each time step.

$$
\begin{gathered}
D_{e} \frac{\partial^{2} S_{w}}{\partial x^{2}{ }_{i}}=\phi \frac{\partial S_{w}}{\partial t} \\
S_{w b} / \Gamma_{j}=S_{f w}(t) \\
S_{\text {winitial }} /{ }_{t=0}=S_{w c}
\end{gathered}
$$

where, $D_{e}$ is effective capillary diffusion coefficient, $S_{w b}$ is boundary water saturation of matrix block, $j$-the $j$-th piece of matrix block boundary, $S_{\text {winitial }}$ is initial water saturation of matrix block.

Eq. (13) is the boundary condition, water saturation on the matrix boundary, which is equal to the saturation in the fracture. Eq. (12) is the initial condition , the distribution of saturation in a single matrix is simplified as average saturation for the purpose of simplification, this initial average saturation is settled equals to $S_{w c}$, which is irreducible water saturation.

In order to solve the Eq. (11-13) and calculate the amount of imbibition on each side of an single matrix. Radial integration boundary element method (RIBEM) [7] is introduced in imbibition problem and is used to calculate target imbibition term. Based on boundary element method (BEM) theory Eq. (11) is changed into its equivalent integral form [8].

$$
\begin{aligned}
& C(\boldsymbol{y}) S_{w}(\boldsymbol{y}, t)=\int_{\Gamma} \frac{G(\boldsymbol{x}, \boldsymbol{y})}{D_{e}} q_{w}(\boldsymbol{x}, t) d \Gamma- \\
& \int_{\Gamma} S_{w}(\boldsymbol{x}, t) \frac{\partial G(\boldsymbol{x}, \boldsymbol{y})}{\partial n_{i}} d \Gamma+\phi \int_{\Omega} G(\boldsymbol{x}, \boldsymbol{y}) \frac{\partial S_{w}(\boldsymbol{x}, t)}{\partial t} d \Omega
\end{aligned}
$$

Here, $\boldsymbol{x}$ is field point. $\boldsymbol{y}$ is source point. $\Gamma$ is boundary of calculation area. $\Omega$ is calculation area. $\boldsymbol{n}_{\mathrm{i}}$ is normal vector of element. $\mathbf{C}(\boldsymbol{y})$ is coefficient which is decided by the position of $\boldsymbol{y}, G(\boldsymbol{x}, \boldsymbol{y})$ is fundamental solution. $q_{\mathrm{w}}$ is the imbibition rate of matrix block, $\mathcal{E}$ is distance between source point $\boldsymbol{y}$ and field point $\boldsymbol{x}$.

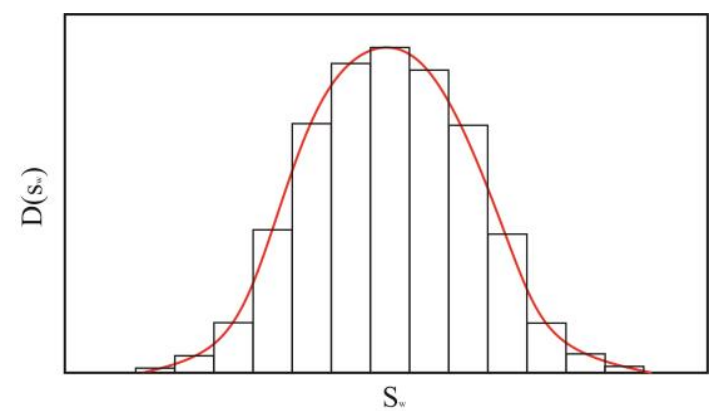

Fig. 4. Schematic diagram of capillary diffusion coefficient.

From Eq. (14), it can be seen clearly that the last term of the right side is difficult to calculate as it is a domain integral, for the assumption mentioned before that water saturation distribution inside of the matrix is replaced by average water saturation and by doing this the domain integration term can be simplified as a integration that only concerns to boundary, the simplified last term in Eq. (11) is listed in Eq. (15), write Eq. (15) into matrix form, change of matrix average saturation can be solved [9].

$$
\begin{gathered}
C_{i} S_{i}=\sum_{j=1}^{N} q_{w b j} \int_{\Gamma_{j}} \frac{G(\boldsymbol{x}, \boldsymbol{y})}{D_{e}} d \Gamma-\sum_{j=1}^{N} S_{w b j} \int_{\Gamma_{j}} \frac{\partial G(\boldsymbol{x}, \boldsymbol{y})}{\partial n_{i}} d \Gamma \\
+\frac{\phi \bar{S}_{w}^{m+1}}{d t} \sum_{j=1}^{N} \int_{\Gamma_{j}} G^{\prime}(\boldsymbol{x}, \boldsymbol{y}) d \Gamma-\frac{\phi \bar{S}_{w}^{m}}{d t} \sum_{j=1}^{N} \int_{\Gamma_{j}} G^{\prime}(\boldsymbol{x}, \boldsymbol{y}) d \Gamma
\end{gathered}
$$

Here, $N$ is number of segment divided by the matrix block boundary. $S_{w \mathrm{bj}}$ is water saturation of the jth segment. $q_{w b j}$ is imbibition rate on the jth segment. $G$ ' is the first derivative of the fundamental solution. $S_{\mathrm{w}}$ is average water saturation of matrix, dimensionless. $m$ stands for time step.

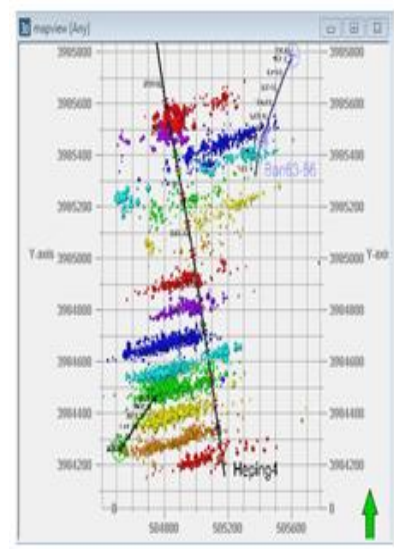

(a)Microsesmic monitoring map

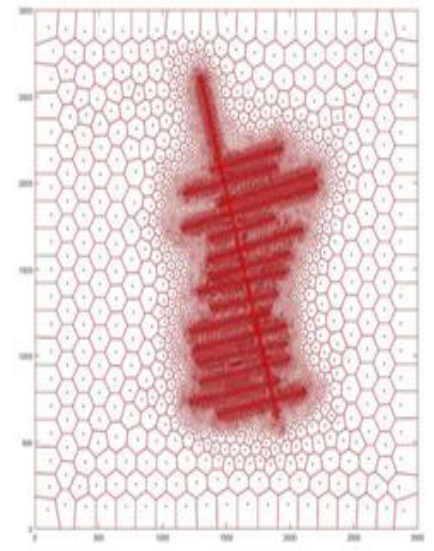

(b)Meshing of fracture network Fig. 5. Fracture network mode.

\section{CASE Study}

In order to figure out the influence of imbibition on real tight reservoir, a real multi-staged volume fracturing horizontal production well case is used, hydraulic fracture distribution is presented in Fig. 5 (a), which is the result of 
micro-seismic monitoring results, Fig. 5 (b) is the corresponding PEBI grid.

TABLE I: PARAMETERS IN THE MODEL

\begin{tabular}{lclc}
\hline \hline Parameters & Value & Parameters & Value \\
\hline $\begin{array}{l}\text { Initial reservoir pressure, } \\
\text { MPa }\end{array}$ & 25 & $\begin{array}{l}\text { Permeability of } \\
\text { hydraulic fractures, } \\
\text { mD }\end{array}$ & 2000 \\
BHP,Mpa & 5 & $\begin{array}{l}\text { Porosity of the } \\
\text { reservoir, fraction }\end{array}$ & 0.2 \\
$\begin{array}{l}\text { Permeability of the tight } \\
\text { matrix reservoir with } \\
\text { boundary layer, mD }\end{array}$ & 1 & $\begin{array}{l}\text { Numbers of } \\
\text { hydraulic fractures }\end{array}$ & 15 \\
$\begin{array}{l}\text { Permeability of the tight } \\
\text { matrix reservoir without } \\
\text { boundary layer, mD }\end{array}$ & 500 & $\begin{array}{l}\text { Porosity of } \\
\text { hydraulic fractures, } \\
\text { fraction }\end{array}$ & 0.5 \\
$\begin{array}{l}\text { Permeability of the nature } \\
\text { fracture reservoir, mD }\end{array}$ & 100 & $\begin{array}{l}\text { Average } \\
\text { half-length of } \\
\text { hydraulic fractures, } \\
\text { m }\end{array}$ & 1500 \\
\hline \hline
\end{tabular}

Parameters used in the simulation model are shown in Table I; the capillary pressure and relative permeability of tight formation with and without boundary layer are shown in Fig. 6.

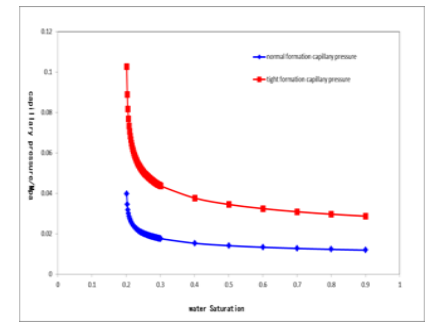

(a)comparison of capilla pressure curve

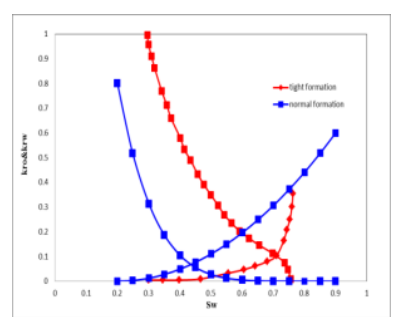

(b) comparison of relative permeability curve
Fig. 6. Comparison of capillary pressure and relative permeability between tight and normal formation.
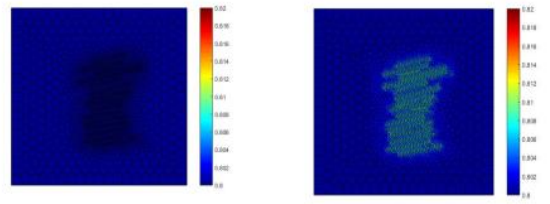

(a) no imbibition (b) with boundary layer

(c) without boundary layer

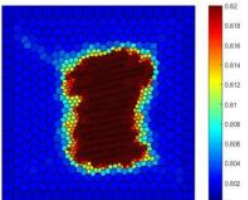

Fig. 7. Water saturation contour map at 200days.

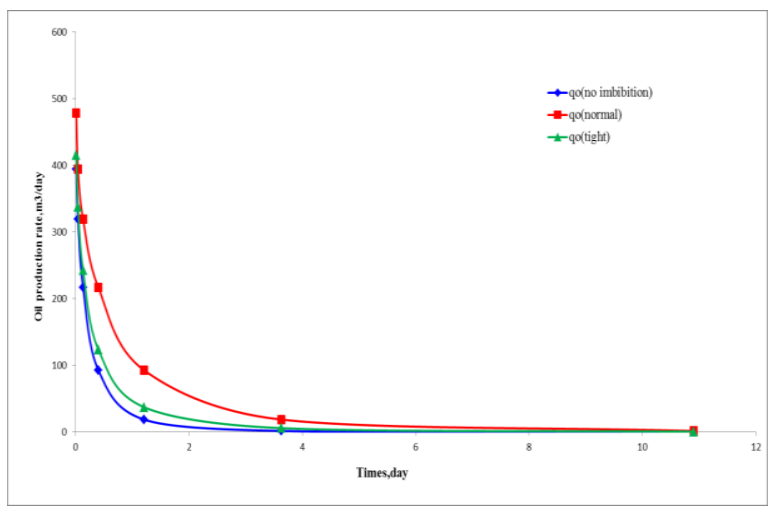

Fig. 8. Oil production rate with different condition.
A total three cases are simulated, the first case simulated without any imbibition, the second case simulated with imbibition in tight reservoir without boundary layer, the third case simulated with imbibition in normal reservoir with boundary layer. 200days exploring processes are simulated, water saturation contour maps at 200day are listed in Fig. 7 and oil production rate are listed in Fig. 8.

\section{CONCLUSION}

(1) this article come up with a complete model to simulate the imbibition effect on any kind of reservoir, the simulate model include meshing strategy and mass transfer method which is able to consider the characteristic of boundary layer.

(2) imbibition effect changes the oil saturation distribution near the wellbore area, The stronger the imbibition ability, the higher the oil saturation near the wellbore area. As for tight oil reservoir, the ability of tight oil reservoir is weak, it's effect on water saturation around the wellbore area is limited.

(3) Imbibition has an effect on oil production rate and the effect is mainly concentrate on early stage of production, compared with formation without boundary layer, imbibition with boundary layer only brings very limited increase on oil production rate. So, for tight reservoir considering boundary layer, imbibition effect is slight, which has no significant effect on improving the oil exploring efficiency.

\section{ACKNOWLEDGMENT}

The authors are grateful to financial support of National 973 Program of China (No.2015CB250902), the National Natural Science Foundation of China (No. 51674273) and the National Natural Science Foundation of China (No. 51574258).

\section{REFERENCES}

[1] P. Jia, L Cheng, S Huang, et al., "A semi-analytical model for the flow behavior of naturally fractured formations with multi-scale fracture networks [J]," Journal of Hydrology, vol. 537, pp. 208-220, 2016.

[2] P. W. Von and L. Ganzer, "Reservoir simulation model for fractured and partially fractured reservoirs based on PEBI grids," presented at the SPE Reservoir Simulation Symposium, Houston, TX, pp. 11-14, February 2001.

[3] R Cao, L Cheng, et al., "A new model for determining the effective permeability of tight formation," Transp Porous Med., vol. 112, pp. 21-37, 2016.

[4] X. Tian, L. Cheng, and Y. Yan, "To estimate relative permeability in tight oil reservoirs," J. Petrol. Explor. Prod. Technol., vol. 5, no. 3, pp 1-10, 2014.

[5] W. R. Purcell, "Capillary pressures - their measurement using mercury and the calculation of permeability therefrom," Pet Trans. AIME., vol. 186, pp. 39-48, 1946.

[6] D. Kashchiev and A. Firoozabadi, "Analytical solutions for 1D counter current imbibition in water-wet media," SPEJ., vol. 8, no. 4, pp. 401-408, 2003.

[7] B. Yu, W. Yao, and X Gao, "A combined approach of RIBEM and precise time integration algorithm for solving transient heat conduction problems," Numer Heat Tr B-Fund, vol. 65, pp. 155-173, 2014.

[8] B. Yu, W. A. Yao, and X. W. Gao, “A time-domain precise BEM for solving transient heat conduction problems with variable hea conductivities," Wit Transactions on Modelling \& Simulation, vol. 56 , pp. 273-282, 2013.

[9] X. W. Gao, "The radial integration method for evaluation of domain integrals with boundary-only discretization," Eng. Anal. Bound. Elem., vol. 26, no. 10, pp. 905-916, 2002 


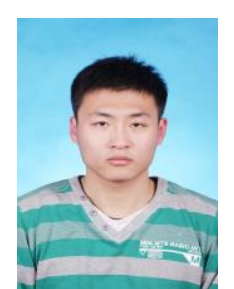

Xu Zhongyi was born in the city of Yili, Xingjiang, China in 1989. Mr. Xu received his master degree from china University of geoscience, Beijing in 2014, at present he is pursing $\mathrm{PhD}$ in oil and gas development engineering from China University of Petroleum, Beijing, his research interests include unconventional oil and gas exploration.

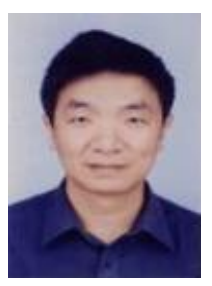

Lin-song Cheng was born in the city of Yingcheng, Hubei, China in 1965. Mr. Cheng received his doctor degree from China University of Petroleum, Beijing in 1994. Now he is the professor and vice president at the Faculty of Petroleum Engineering, China University of Petroleum, Beijing. His research directions include thermal recovery, mechanics of flow through porous media, the recovery method for low-permeability reservoir.

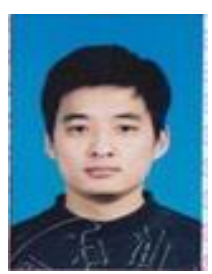

Renyi Cao was born in the city of Weifang Shandong, China in 1981. Mr. Cao received his doctor degree from China University of Petroleum, Beijing in 2009. Now he is the vice professor at the Faculty of Peum Engineering, China University of Petroleum, Beijing. His research directions include mechanics of flow through porous media, the recovery method for low-permeability reservoir.

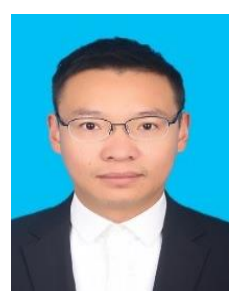

Sidong Fang was born in the city of nanchong Sichuan, China in 1988. Dr. Fang received his bachelor's degree from Southwest Petroleum University in 2011. At present he is pursing $\mathrm{PhD}$ in oil and gas development engineering from China University of Petroleum, Beijing, his research interests include unconventional oil exploration.

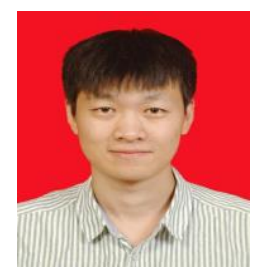

Jianlong Liang was born in the city of Jingzhou, Hubei, China in 1988. Mr. Liang received his master degree in 2014 from china University of geoscience, Beijing. At present he is a researcher at Great wall Drilling company. His research interests include unconventional oil and gas exploration. 